\title{
Effect of Listerine Mouthwash with Green Tea on the Inhibition of Streptococcus Mutans: A Microbiologic Study
}

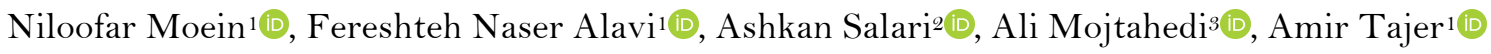

\begin{abstract}
'Department of Operative Dentistry, School of Dentistry, Guilan University of Medical Sciences, Rasht, Iran. ${ }^{2}$ Department of Periodontics, School of Dentistry, Guilan University of Medical Sciences, Rasht, Iran. ${ }^{3}$ Department of Microbiology, Guilan University of Medical Sciences, Rasht, Iran.
\end{abstract}

Author to whom correspondence should be addressed: Fereshteh Naser Alavi, Guilan University of Medical Science, School of Dentistry, Lacan Road, Gas Square, Rasht, Iran. Phone: +98013 3348416. E-mail: minanasseralavi@yahoo.com.

Academic Editors: Alessandro Leite Cavalcanti and Wilton Wilney Nascimento Padilha

Received: 27 December 2019 / Accepted: 11 May 2020 / Published: 10 June 2020

How to cite this article: Moein N, Alavi FN, Salari A, Mojtahedi A, Tajer A. Effect of listerine mouthwash with green tea on the inhibition of streptococcus mutans: a microbiologic study. Pesqui Bras Odontopediatria Clín Integr. 2020; $20: e 5477$. https://doi.org/10.1590/pboci.2020.106

\begin{abstract}
Objective: To evaluate in vitro the antimicrobial effect of Listerine-green tea mouthwash on Streptococcus mutans (SM) in comparison with $0.12 \%$ Chlorhexidine (CHX) and Listerine-Zero. Material and Methods: The sensitivity and growth inhibition of SM bacterial species were evaluated and compared between Listerine-green tea, $0.12 \%$ CHX and Listerine-Zero mouthwashes. Sixty plates containing SM colonies were prepared in three groups $(n=20)$, and growth inhibition zones were measured using the disk diffusion agar test in $\mathrm{mm}$. Data were analyzed with SPSS 21. One-way ANOVA was used to compare the efficacy of the three mouthwashes tested. Post hoc Tukey tests were used for two-by-two comparisons. Statistical significance was defined at $\mathrm{P}<0.05$. Results: Analysis of data showed significant differences between the three groups $(\mathrm{p}<0.001)$; $0.12 \%$ CHX was the most effective mouthwash, and Listerine-Zero exhibited the least effect on the growth inhibition of SM $(p<0.004)$. Conclusion: All three mouthwashes were significantly effective in inhibiting the growth of SM. The effect of Listerine-green tea mouthwash was higher than that of Listerine-Zero and less than that of $0.12 \% \mathrm{CHX}$.
\end{abstract}

Keywords: Biomedical and Dental Materials; Mouthwashes; Microbiological Techniques. 


\section{Introduction}

Dental caries is still considered a major health problem in many countries [1]. The condition is the result of an interaction between specific bacteria and diet on the one hand and the tooth surface plaque. Of all the bacterial species, Streptococcus mutans (SM) has been reported as the principal etiologic agent for dental caries. SM is an anaerobic gram-positive coccus bacterial species that forms a large amount of extracellular polysaccharides using dietary sucrose and is the principal etiologic agent involved in the formation of cariogenic plaque [2].

Currently, there are great concerns about the prevention and preventive care for dental caries at home [3]. The use of mechanical techniques to preserve the oral health, including brushing and use of dental floss, is the focus of dentists' attention to decreasing the counts of microorganisms, especially SM, and provide caries prevention care. Use of antimicrobial agents (chemical and herbal) has been recommended to improve the efficacy of mechanical methods [4]. Mouthwashes are safe and effective antimicrobial and anti-plaque agents that prevent adhesion, colonization, metabolic activity and proliferation of bacteria [5]. It is difficult to decide on the use of a specific mouthwash for a specific aim due to diversities in the antibacterial efficacy, cytotoxicity and kinetics of different solutions [6].

Of all the commonly used mouthwashes, chlorhexidine (CHX) digluconate has a long history as an effective antimicrobial agent in inhibiting the formation of dental plaque. CHX is considered a gold standard in decreasing the counts of oral microbial counts. In many studies, it is considered a positive control group to compare its effect with that of other materials. However, despite all its advantages, it has some disadvantages, including a change in taste perception, tooth discoloration and xerostomia [7].

Studies on the use of Listerine mouthwash have shown its high antimicrobial activity against oral microorganisms [8]. Its antiseptic properties are attributed to its content of active antibacterial agents, including essential oils [9].

Currently, the use of herbal medicines with antibacterial properties has increased for the treatment of infections. Recent in vitro, animal and human studies have reported the positive effect of green tea on decreasing the rate of dental caries [10,11]. Green tea is one of the traditional drinks in China and Japan, which is derived from the young stems of Camellia sinensis [12].

One of the new achievements in the treatment of dental caries is the use of a combination of several antibacterial agents. Recently, Listerine mouthwash has been marketed, with green tea as one of its constituents, and the manufacturer claims that adding green tea with antibacterial properties and the polyphenols in its structure has increased the inhibitory effects of this mouthwash on the initiation of caries.

Considering the high prevalence of caries in different communities, its prevention is one of the research priorities. In addition, due to the diversity of the mouthwashes that are available and the current data, this in vitro study was designed to evaluate the inhibitory effect of Listerine containing green tea and Listerine-Zero. In addition, $0.12 \%$ CHX mouthwash was used, which is the gold standard mouthwash in the treatment of infections, to compare the results and effects on SM.

\section{Material and Methods}

Study Design

In the present in vitro study, the standard strain of SM (PTCC 1683) was used to evaluate the inhibitory effect of Listerine-green tea mouthwash (Listerine, Johnson \& Johnson S.p.A, Italy) and compare it with Listerine Zero (Listerine, Johnson \& Johnson S.p.A, Italy) and 0.12\% CHX (Behasa, Iran). 


\section{Experimental Stage}

The microbial inoculation technique was used to carry out the antibiogram test with the Kirby-Bauer standard method or disk diffusion test, commonly used to identify a microorganism that is resistant or sensitive to a specific antibiotic. A sterile loop was used to transfer some isolated colonies of SM standard strain from the culture medium into a test tube containing sterile physiologic serum and incubated at $37^{\circ} \mathrm{C}$ for 2-3 hours. Then the turbidity of the test tubes was compared with 0.5 standard McFarland concentration because the amount of microbes inoculated affects the growth inhibition zone in the disk diffusion test. The turbidity of this test tube is almost equal to $1.5 \times 108$ bacteria/mL. After achieving suspension turbidity equal to that of $0.5 \mathrm{McF}$ arland concentration tube, a sterile swab was used to remove some bacterial suspension and inoculate on 60 plates containing Mueller-Hinton agar. The plates were randomly divided into three groups $(n=20)$ to evaluate the performance of the mouthwashes: Group 1: Listerine-green tea; Group 2: Listeria-Zero; and Group 3: 0.12\% CHX.

A blank disk (Padtan Teb, Iran), containing Listerine-green tea, and disks containing 0.12\% CHX and Listerine Zero mouthwashes, were dried in an incubator at $37^{\circ} \mathrm{C}$ for 1 hour. Then they were placed on the prepared culture medium using the microbial method. One specific microbial culture plate was allocated to each disk because the effect of each mouthwash and the diameter of the halo of each disk were unknown.

After 24 hours of incubation of the plates, the disks containing the mouthwashes were evaluated by determining the size of the microbial growth inhibition zone. The diameter of the SM growth inhibition zone around each disk was measured with a ruler in $\mathrm{mm}$. The mean values were determined in each group, and the bactericidal effects of the three mouthwashes were evaluated and compared.

\section{Data Analysis}

Data were analyzed with SPSS Statistics for Windows Software (IBM Corp., Armonk, NY, USA), version 21. Descriptive statistics was used to calculate the mean and standard deviation. One-way ANOVA and post hoc Tukey tests were used. Statistical significance was set at $\mathrm{P}<0.05$.

Ethical Aspects

This research was approved by the Research Committee of the Guilan University of Medical Sciences (IR.GUMS.REC.1397.355).

\section{Results}

The normal distribution of data was confirmed by the Kolmogorov-Smirnov test. Table 1 presents the diameters of SM growth inhibition zones in the three study groups. One-way ANOVA showed significant differences between the three groups $(\mathrm{p}<0.001)$. Two-by-two comparisons of the groups with post hoc Tukey tests showed that the effect of CHX was significantly higher than those of Listerine-green tea and Listerine Zero $(\mathrm{p}<0.001$ and $\mathrm{p}<0.001)$ and Listerine-green tea was significantly higher than that of Listerine-Zero $(\mathrm{p}<0001)$.

Table 1. The mean diameters of SM growth inhibition zones.

\begin{tabular}{cc}
\hline Groups & Mean $(\mathbf{S D})$ \\
\hline Listerine Green Tea & $21.43(3.76)^{\mathrm{A}}$ \\
Listerine Zero & $18.73(8.91)^{\mathrm{B}}$ \\
Chlorhexidine & $26.35(1.34)^{\mathrm{C}}$ \\
\hline The differences between dissimilar letters are significant $(\mathrm{p}<0.05)$.
\end{tabular}

The differences between dissimilar letters are significant $(\mathrm{p}<0.05)$. 


\section{Discussion}

Streptococcus mutans is the chief etiologic agent for dental caries. It can adhere to the acquired pellicle as the first step in plaque formation. As a result, the elimination of this bacterial species prevents plaque formation and development of caries. The mechanical methods of plaque inhibition have some limitations; therefore, chemical techniques of dental plaque inhibition have been recommended. The use of disinfecting mouthwashes can be helpful for decreasing dental plaque [13]. The present study evaluated the effect of Listerine-green tea mouthwash on the inhibition of SM and compared it with the efficacy of $0.12 \%$ CHX and Listerine-Zero mouthwashes.

Based on the results, all the three mouthwashes exhibited the ability to inhibit the proliferation of SM, with 0.12 CHX exhibiting the highest inhibitory effect. Many studies on oral microorganisms have reported that CHX is the best mouthwash [14,15], deeming it the gold standard in the chemical treatment against SM and dental caries [1]. Consistent with the present study, other authors showed that CHX-containing mouthwashes ( $0.2 \%$ and $0.06 \%$ concentrates) and Total Care Listerine Zero inhibited the formation of plaque by different species of Streptococci [16]. They also reported that CHX-containing mouthwashes were more effective than Total Care Listerine Zero mouthwash. These mouthwashes effectively inhibited the initiation of dental caries, and they decreased colonization of pathologic bacterial species and also exerted no destructive effects on innocent bacterial species competing with SM.

The positive effect of $0.12 \%$ CHX mouthwash on decreasing the number of SM and Lactobacillus colonies has been described in the literature [17]. CHX is a bisguanide and is adsorbed to the surface of tooth pellicle, plaque and mucosa, and its cationic nature increases its adsorption to the surfaces mentioned above. The antibacterial activity of this mouthwash is the result of its absorption by extracellular polysaccharides [18].

On the other hand, contrary to the results of the present study, previous authors showed the better effect of Listerine mouthwash on planktonic and biofilm bacteria compared to 0.0005\% CHX (diluted) [9]. In another research, $12 \% \mathrm{CHX}$ was more effective in decreasing plaque and gingivitis; however, the use of diluted CHX $(0.0005 \%)$ did not exhibit any antibacterial activity [19]. It appears that the concentration of CHX has an important role in its antimicrobial activity.

Another finding of the present study was the better and higher antibacterial effect of Listerine-green tea mouthwash compared to that of Listerine-Zero mouthwash. Listerine contains active ingredients, including essential oils, and its antimicrobial activity against oral microorganisms has already been evaluated and confirmed $[8]$.

Recently, Listerine-green tea mouthwash has been produced and marketed by adding green tea to the main mouthwash. A large number of studies have evaluated the antimicrobial, anti-inflammatory and anticariogenic effects of green tea extract [10,11]; however, no study is available on the evaluation and comparison of the effect of this new mouthwash on SM.

Green tea is produced from the fresh leaves of Camellia sinensis and contains polyphenols, especially flavonoids. The principle flavonoids in tea are epigallocatechin-3-gallate and epicatechin [10]. The anticariogenic activity of catechin might be attributed to its direct anti bactericidal effect on SM, inhibition of the adhesion of bacteria to tooth surfaces and inhibition of bacterial amylase. Drinking green tea cleans the oral cavity and the individuals drinking large amounts of green tea are affected by dental caries at a lower rate [20]. Use of a combination of several antibacterial agents in one product might be one of the methods to increase its anti-plaque efficacy [17]. Considering the confirmation of the effects of green tea extract and 
Listerine mouthwashes in different studies on the inhibition of SM, it is possible to justify the higher antibacterial effect of Listerine-green tea mouthwash compared to Listerine-Zero.

Consistent with the present study, the positive effect of $0.5 \%$ green tea extract on decreasing the colony counts of SM and Lactobacillus was previously demonstrated [17]; however, it was less effective than $0.12 \%$ CHX mouthwash. Contrary to the present research, a study showed that the use of green tea extract mouthwash can decrease the oral microorganism counts similar to CHX, and the two mouthwashes had similar effects [21]. The differences in the results of studies might be attributed to the differences in the concentration of active agents in the formulation of the mouthwash. Unfortunately, the concentration of the green tea used in Listerine mouthwash has not been reported by the manufacturer, and it is possible that a similar concentration has not been used in studies.

Previous authors evaluated the effect of EGCG-S (epigallocatechin-3-gallate-stearate polyphenol) on SM biofilm and showed that EGCG-S solutions at $200 \mathrm{mg} / \mathrm{mL}$ concentration at 4 hours and $250 \mathrm{mg} / \mathrm{mL}$ concentration at 2-4 hours completely inhibited streptococcal biofilm formation, and its efficacy was concentration-dependent [22]. In addition, the authors reported that EGCG-S of green tea at higher concentrations was able to completely inhibit the growth of S. mutans, similar to the effect of $0.1 \% \mathrm{CHX}$. Overall, in the present study, the $0.12 \%$ concentration of CHX exhibited the highest antibacterial properties; however, the use of CHX, apart from its strong antibacterial effects, has some disadvantages, including tooth discoloration, loss of the sense of taste and allergic reactions. For this reason, sometimes its diluted concentration is recommended. However, this may decrease antibacterial efficacy [9,19].

Therefore, given the presence of nature components in Listerine-green tea mouthwash and lack of tooth discoloration potential in this mouthwash, it might be a proper alternative for CHX for daily use.

\section{Conclusion}

Listerine mouthwash containing green tea was more effective than Listerine Zero in inhibiting S. mutans proliferation. However, both mouthwashes were less effective than $0.12 \% \mathrm{CHX}$ mouthwash.

\section{Authors' Contributions}

\begin{tabular}{|c|c|c|}
\hline NM & (iD) $0000-0001-5084-7021$ & $\begin{array}{l}\text { Conceptualization, Methodology, Formal Analysis and Writing - Original Draft } \\
\text { Preparation }\end{array}$ \\
\hline FNA & (iD) $0000-0002-4879-5434$ & $\begin{array}{l}\text { Conceptualization, Methodology, Formal Analysis, Writing - Original Draft } \\
\text { Preparation and Writing - Review and Editing. }\end{array}$ \\
\hline AS & (iD) $0000-0003-4504-5141$ & Conceptualization, Methodology and Writing - Original Draft Preparation. \\
\hline AM & (iD) $0000-0002-5385-9367$ & $\begin{array}{l}\text { Conceptualization, Methodology, Investigation and Writing - Original Draft } \\
\text { Preparation. }\end{array}$ \\
\hline AT & Do00-0001-9525-609X & Investigation, Formal Analysis and Writing - Original Draft Preparation. \\
\hline
\end{tabular}

\section{Financial Support}

None.

\section{Conflict of Interest}

The authors declare no conflicts of interest.

\section{References}


[1] Padiyar B, Marwah N, Gupta S, Padiyar N. Comparative evaluation of effects of triphala, garlic extracts, and chlorhexidine mouthwashes on salivary Streptococcus mutans counts and oral hygiene status. Int J Clin Pediatr Dent 2018; 11(4):299-306. https://doi.org/10.5005/jp-journals-10005-1530

[2] Hamada S, Slade HD. Biology, immunology, and cariogenicity of Streptococcus mutans. Microbiol Rev 1980; 44(2):331-84.

[3] Filoche SK, Soma D, van Bekkum M, Sissons CH. Plaques from different individuals yield different microbiota responses to oral-antiseptic treatment. FEMS Immunol Med Microbiol 2008; 54(1):27-36. https://doi.org/10.1111/j.1574-695X.2008.00443.x

[4] Groppo FC, Ramacciato JC, Simoes RP, Florio FM, Sartoratto A. Antimicrobial activity of garlic, tea tree oil, and chlorhexidine against oral microorganisms. Int Dent J 2002; 52(6):433-7. https://doi.org/10.1111/j.1875-595x.2002.tbo0638.x

[5] Sundas S, Rao A. Comparative evaluation of chlorhexidine and sodium fluoride mouthwashes on Streptococcus mutans. J Nepal Dent Assoc 2011; 12(1):17-21.

[6] Erciyas AF, Erciyas K, Sarıkaya R. Genotoxicity of two mouth-wash products in the drosophila wing-spot test. Food Chem Toxi-col 2010; 48(10): 2577-80. https://doi.org/10.1016/j.fct.2010.06.004

[7] Hassan SM, Mobarak EH, Fawzi EM. The efficacy of different regimens of chlorhexidine as an antimicrobial agent for a group of Egyptians. J Egypt Public Health Assoc 2008; 83(5-6):435-50.

[8] Pan P, Barnett ML, Coelho J, Brogdon C, Finnegan MB. Determination of the in situ bactericidal activity of an essential oil mouthrinse using a vital stain method. J Clin Periodontol 2000; 27(4):256-61. https://doi.org/10.1034/j.1600-051x.2000.027004256.x

[9] Oyanagi T, Tagami J, Matin K. Potentials of mouthwashes in disinfecting cariogenic bacteria and biofilms leading to inhibition of caries. Open Dent J 2012; 6:23-30. https://doi.org/10.2174/1874210601206010023

[10] Subramaniam P, Eswara U, Maheshwar Reddy KR. Effect of different types of tea on Streptococcus mutans: An in vitro study. Indian J Dent Res 2012; 23(1):43-8. https://doi.org/10.4103/0970-9290.99037

[11] Ardakani MR, Golmohammadi S, Ayremlou S, Taheri S, Daneshvar S, Meimandi M. Antibacterial effect of Iranian green-tea-containing mouthrinse vs. chlorhexidine 0.2\%: an in vitro study. Oral Health Prev Dent 2014; 12(2):15762. https://doi.org/10.3290/j.ohpd.a31663

[12] Chatterjee P, Chandra S, Dey P, Bhattacharya S. Evaluation of anti-inflammatory effect of green tea and black tea: A comparative in vitro study. J Adv Pharm Technol Res 2012; 3(2):136-8. https://doi.org/10.4103/223 1-4040.97298

[13] Chitsazi M, Shirmohammadi A, Balayi E. Effect of herbal and chemical mouth-rinses on periodontal indices; comparison of matrica, persica and chlorhexidine. J Dent Shiraz Univ Med Sci 2008; 8(4):54-60.

[14] Shen Y, Stojicic S, Haapasalo M. Antimicrobial efficacy of chlorhexidine against bacteria in biofilms at different stages of development. J Endod 2011; 37(5):657-61. https://doi.org/10.1016/j.joen.2011.02.007

[15] Rohrer N, Widmer AF, Waltimo T, Kulik EM, Weiger R, Filipuzzi-Jenny E, et al. Antimicrobial efficacy of 3 oral antiseptics containing octenidine, polyhexamethylene biguanide, or Citroxx: can chlorhexidine be replaced? Infect Control Hosp Epidemiol 2010; 31(7):733-9. https://doi.org/10.1086/653822

[16] Ardizzoni A, Pericolini E, Paulone S, Orsi CF, Castagnoli A, Oliva I, et al. In vitro effects of commercial mouthwashes on several virulence traits of Candida albicans, viridans streptococci and Enterococcus faecalis colonizing the oral cavity. PLoS One 2018; 13(11):e0207262. https://doi.org/10.1371/journal.pone.0207262

[17] Hegde RJ, Kamath S. Comparison of the Streptococcus mutans and Lactobacillus colony count changes in saliva following chlorhexidine $(0.12 \%)$ mouth rinse, combination mouth rinse, and green tea extract $(0.5 \%)$ mouth rinse in children. J Indian Soc Pedod Prev Dent 2017; 35(2):150-5. https://doi.org/10.4103/JISPPD.JISPPD_13_17

[18] McBain AJ, Bartolo RG, Catrenich CE, Charbonneau D, Ledder RG, Gilbert P. Effects of a chlorhexidine gluconatecontaining mouthwash on the vitality and antimicrobial susceptibility of in vitro oral bacterial ecosystems. Appl Environ Microbiol 2003; 69(8):4770-6. https://doi.org/10.1128/aem.69.8.4770-4776.2003

[19] Sekino S, Ramberg P. The effect of a mouth rinse containing phenolic compounds on plaque formation and developing gingivitis. J Clin Periodontol 2005; 32(10):1083-8. https://doi.org/10.1111/j.1600-051X.2005.00793.x

[20] Hamilton-Miller JMT. Anti-cariogenic properties of tea (Camellia sinensis). J Med Microbiol 2001; 50(4):299-302. https://doi.org/10.1099/0022-1317-50-4-299

[21] Moghbel AAH, Farajzadeh SA, Aghel N, Raisi N. Formulation and evaluation of green tea antibacterial mouthwash effect on the aerobic mouth bacterial load. Jundishapur Sci Med J 2010; 9(4):317-30.

[22] Melok AL, Lee LH, Mohamed Yussof SA, Chu T. Green tea polyphenol epigallocatechin-3-gallate-stearate inhibits the growth of Streptococcus mutans: a promising new approach in caries prevention. Dent J 2018; 6(3):38. https://doi.org/10.3390/dj6030038 